\title{
Sustentabilidade de sistemas de rotação e sucessão de culturas em solos de várzea no Sul do Brasil
}

\author{
Crop rotations sustainability and successions systems in tilled plain areas in Southern Brazil
}

\author{
Francisco de Jesus Vernetti Junior ${ }^{*}$ Algenor da Silva Gomes ${ }^{\mathrm{I}}$ \\ Luis Osmar Braga Schuch"I
}

\section{RESUMO}

A avaliação quantitativa com diferentes tipos de indicadores é fundamental na determinação da sustentabilidade dos sistemas de rotação e sucessão de culturas. Este estudo teve como objetivo avaliar a sustentabilidade de algumas sucessões de culturas em solo de várzea, nos sistemas de plantio direto (PD) e convencional (SC). O trabalho foi conduzido em Pelotas, Rio Grande do Sul (RS), em área experimental da Embrapa Clima Temperado. Os tratamentos foram constituídos por cinco anos de implantação de sistemas de rotação $e$ sucessão de culturas de inverno e de primavera-verão, seguidos de dois anos de pousio e três anos da cultura do arroz irrigado. As culturas de primavera-verão foram o milho e a soja, além do arroz; enquanto que as de inverno foram gramíneas, leguminosas, consórcios, nabo forrageiro e campo natural. $O$ delineamento experimental foi o de blocos ao acaso com parcelas subdivididas, com três repetições. As culturas de verão ocuparam as parcelas, enquanto as espécies de inverno foram alocadas nas subparcelas. Os parâmetros observados foram transformados em índices para padronizar as diversas variáveis, cada uma em relação àquela de melhor comportamento mensurado, e dispostos em quatro categorias de análise: (a) indicadores agronômicos (matéria seca e rendimento de grãos); (b) indicadores ambientais (fertilidade do solo); (c) indicadores energéticos (produção e eficiência energética); d) indicadores econômicos (valor bruto da produção e rentabilidade). Pela média harmônica obtida entre os índices dos indicadores, foram comparados os diferentes sistemas de rotação e sucessão de culturas, inferindo-se daí sua sustentabilidade e suas diferenças (Teste $t P \leq 0,05$ ). Concluiu-se que: (a) todos os sistemas de rotação e sucessão de culturas dos quais o milho faz parte apresentam maior índice de sustentabilidade; (b) os sistemas de rotação e sucessão de culturas $S_{1}$ [gramínea $x$ soja $x$ arroz $(P D)$ ] e $S_{4}$ [nabo x soja $x$ arroz (PD)] apresentam, respectivamente, a maior e a menor sustentabilidade entre aqueles sistemas em que a cultura da soja participa; (c) o sistema $S_{5}$ [campo nativo $x$ soja $x$ arroz
(SC)] apresenta o menor índice de sustentabilidade; (d) o sistema $S_{8}$ [Consórcio x milho x arroz $(P D)$ ] apresenta a melhor distribuição e o melhor equilíbrio entre as diversas perspectivas de sustentabilidade consideradas; (e) o sistema $S_{10}$ [campo nativo $x$ milho $x$ arroz (SC)] tem pior desempenho, no que se refere à sustentabilidade entre os que incluem o milho; $(f)$ o sistema de PD confere maior sustentabilidade às sucessões de cultura.

Palavras-chave: arroz, soja, milho, forrageiras de inverno, qualidade ambiental, indicadores de sustentabilidade, rotação.

\section{ABSTRACT}

The quantitative analysis with different types of indicators is crucial in determining the sustainability of crop succession. This study aimed to appraise that in tilled plain areas. The research was carried out in Pelotas, RS, Brazil at Embrapa Clima Temperado research area. The treatments consisted of successions of winter and spring-summer crops species for five years, followed by two years of fallow and three years of rice cropping. The spring-summer crops were corn and soybean, besides rice; the winter crops were cereals, leguminous pastures, mixtures species, turnip and native grass species. Seeding was performed either by no-till (NT) or conventional (CT). The experiment was planned in a split-plot randomized blocks design with three replications, where the summer crops occupied the plots, while the winter's species were allocated to the subplots. The parameters measured were transformed into index to standardize the variables, each one in relation to the better measurement behavior, and arranged in four categories of analysis: (a) Agronomics indices (dry weight and grain yield); (b) Environmental indicators (soil fertility); (c) Energy Indicators (gain and energy efficiency); d) Economic Indicators (profitability and gross value of production). Through harmonic average rates calculated for each indicator, comparisons to the crop succession were then performed, and its sustainability inferred. Analyses of contrasts

'Embrapa Clima Temperado, Br 392, km 78, CP 403, 96001-970, Pelotas, RS, Brasil. E-mail: vernetti@cpact.embrapa.br. *Autor para correspondência.

IFFaculdade Agronomia Eliceu Elizeu Maciel (FAEM), Universidade Federal de Pelotas (UFPel), Pelotas, RS, Brasil. 
between the crop successions were accomplished too. The results provided the following conclusions: (a) All crop succession which had maize participation had a higher index sustainability; (b) the crops successions $S_{1}$ [Cereals $x$ soybean $x$ rice $(N T)]$ and $S_{4}[$ Turnip $x$ soybean $x$ rice $(N T)]$ had, respectively, the highest and the lowest sustainability among the ones with the soybean crop participation; (c) $S_{5}$ [Native grass $x$ soybean $x$ rice $(C T)]$ showed the lowest sustainability level, (d) $S_{8}$ [Species mixtures $x$ corn $x$ rice $(N T)]$ presented the best distribution and balance between the different classes of sustainability considered; (e) $S_{10}$ [Native grass $x$ corn $x$ rice $(C T)$ ] has the worst performance, regarding the sustainability of the one's which included maize; ( $f$ ) the NT system gives greater sustainability to the crop succession.

Key words: rice, soybean, maize, winter crops (cereals, leguminous pastures, turnip), environment quality, sustainable indicators, crop rotation.

\section{INTRODUÇÃO}

Nos últimos anos, o consumo mundial de cereais tem sido sempre superior à produção, com um crescimento de $8 \%$ entre 2000 e 2006, levando à redução dos estoques mundiais, que atingiram sua menor marca neste último ano (ROYAL SOCIETY OF CHEMISTRY, 2009). Essa mesma fonte informa que o Banco Mundial, por sua vez, estima que um hectare de terra deva alimentar cinco pessoas em 2025, enquanto que, em 1960, essa mesma área alimentava apenas duas pessoas.

No Rio Grande do Sul, os solos de várzea cobrem uma área de 5,4 milhões de hectares (PINTO et al., 1999). Uma das principais atividades econômicas conduzidas nessas áreas tem sido a pecuária extensiva de corte e a cultura do arroz irrigado. Entretanto, a partir do início dos anos 90, surgiram sistemas alternativos, como a utilização do plantio direto para o arroz irrigado e o uso de rotação de culturas, tecnologias capazes de incrementarem a produção de grãos nessas áreas. Esse incremento deve ser apreciado considerando que "o desenvolvimento sustentável é aquele que satisfaz as necessidades do presente sem comprometer as necessidades das futuras gerações”. A sustentabilidade exige abordagens inter e multidisciplinares e, consequentemente, uma maior aproximação entre uma série de disciplinas.

A sustentabilidade de um determinado agroecossistema subordina-se à ação de fatores extrínsecos (fenômenos naturais) e ao manejo dos fatores intrínsecos ao sistema, os quais podem sofrer alterações antrópicas. Várias abordagens têm sido utilizadas nas últimas décadas para minimizar os ambientalmente prejudiciais efeitos da produção agrícola. Tecnologias, tais como plantio direto e o uso de cobertura do solo, são importantes. Essas técnicas reduzem a demanda por energia e água ao reduzir a evaporação, aumentam o teor de carbono do solo, melhoram a estrutura do solo, aumentam as populações de minhoca e combatem a erosão eólica e hídrica.

Diferentes metodologias e indicadores têm sido utilizados para estimativa da sustentabilidade em ecossistemas agrícolas. Para LAL (1999), devem ser utilizadas medidas de produtividade, envolvendo a relação custo $x$ benefício, enquanto que, para CAMPOS \& CAMPOS (2004), devem ser utilizados dados referentes ao consumo e à eficiência energética. Para GOMES et al. (2006), a avaliação quantitativa da qualidade do solo é fundamental para se aferir a sustentabilidade dos sistemas de manejo utilizados.

É possível identificar-se sustentabilidade num sistema de produção agrícola sempre que ele apresentar balanço energético positivo, demonstrar condição de explorar os recursos naturais e produtividade por longo prazo, apresentar condição de manter e/ou melhorar o ambiente com o mínimo de impactos negativos, otimizar a produção com redução do uso de insumos externos, garantir a equidade social, satisfazer as necessidades humanas de alimentos e de renda e atender as necessidades sociais dos agricultores envolvidos (EHLERS, 1999; MASERA et al., 1999).

A identificação de sistemas de manejo do solo baseados em sistemas de culturas com alto aporte concomitante de carbono e nitrogênio ao solo, especialmente via fixação simbiótica e com mínimo revolvimento, aliada a programas de adubação adequados econômica e ambientalmente, é fundamental para a melhoria da qualidade do solo e para a sustentabilidade da diversificação de culturas nas áreas de várzea de clima temperado (GOMES et al., 2006).

A rotação e/ou sucessão de culturas em solos de várzea cultivados com arroz irrigado visa a diminuir os níveis de infestação de plantas daninhas nas lavouras de arroz, principalmente do arrozvermelho, melhorar o uso do solo e sua qualidade, otimizar o uso das máquinas e da mão-de-obra, diversificar a renda, romper ciclos de doenças e pragas e aumentar a rentabilidade da área (VERNETTI JÚNIOR et al., 2003).

A utilização do sistema plantio direto (PD), em culturas alternativas ao arroz irrigado, é recente e está associada à expansão da rotação/sucessão de culturas nessas áreas. A escolha de culturas alternativas de valor econômico, como, por exemplo, a soja, pode contribuir para manter a competitividade do sistema. Segundo VERNETTI JR. et al. (2002), no agroecossistema de solos de várzea, o rendimento de grãos, tanto da soja, como do milho, obtidos em PD, foi maior que o obtido no sistema convencional (SC). 
O cultivo de forrageiras de inverno nesse agroecossistema pode contribuir na forma de restos vegetais mantidos na superfície do solo para a diminuição da ocorrência de invasoras, a manutenção da umidade do solo e o incremento de matéria orgânica, além de proporcionar benefícios para culturas subsequentes, como, por exemplo, o da diminuição da necessidade de aplicação de fertilizantes, decorrente do aproveitamento da adubação aplicada anteriormente.

Outra prática que contribui para a viabilização econômica das propriedades rurais e para o próprio plantio direto é a integração agriculturapecuária. Dentro desse conceito, as áreas de lavouras dão suporte à pecuária via produção de alimentação animal, seja na forma de grãos, silagem e feno, seja na forma de pastejo direto; e aumentam a capacidade de suporte da propriedade, propiciando a venda de animais na entressafra e a melhor distribuição de receita durante o ano.

A análise energética pode fornecer uma das poucas medidas capazes de permitir uma análise comparativa confiável entre sistemas agrícolas (BAILEY et al., 2003). Estimativas dos balanços de energia e de eficiência energética são importantes instrumentos no monitoramento da agricultura, ante o uso de fontes de energia não renováveis. Em função do exposto, procurou-se avaliar a sustentabilidade de alguns sistemas de rotação e sucessão de culturas envolvendo forrageiras de inverno, soja, milho e arroz em solo de várzea, nos sistemas de plantio direto e convencional.

\section{MATERIAL E MÉTODOS}

O trabalho de campo foi conduzido em Pelotas, Rio Grande do Sul (RS), em área experimental da Embrapa Clima Temperado (3152'00'’s, 52²1’24’W), em um Planossolo Háplico eutrófico solódico. Os tratamentos foram constituídos por sucessões de forrageiras de inverno e culturas de verão por cinco anos, seguidos por dois anos de pousio e três anos de cultivo do arroz irrigado. Na primeira fase, utilizaram-se, no verão, as culturas do milho e da soja, semeadas em plantio direto (PD), em sucessão a gramíneas, leguminosas, consórcios (gramínea + leguminosa), nabo-forrageiro e em semeadura convencional (SC). Dessa forma, os sistemas de rotação e sucessão de culturas estudadas foram: $\mathrm{S}_{1}$ - gramínea $\mathrm{x}$ soja x arroz (PD); $\mathrm{S}_{2}$ - leguminosa x soja x arroz (PD); $\mathrm{S}_{3}$ - consórcio $\mathrm{x}$ soja $\mathrm{x}$ arroz (PD); $\mathrm{S}_{4}$ - nabo $\mathrm{x}$ soja $\mathrm{x}$ arroz (PD); $\mathrm{S}_{5}$ - campo nativo x soja x arroz (SC); $\mathrm{S}_{6}$ - gramínea $\mathrm{x}$ milho $\mathrm{x}$ arroz $(\mathrm{PD}) ; \mathrm{S}_{7}$ - leguminosa $\mathrm{x}$ milho $\mathrm{x}$ arroz
(PD); $\mathrm{S}_{8}$ - consórcio x milho x arroz (PD); $\mathrm{S}_{9}$ - nabo $\mathrm{x}$ milho $x$ arroz (PD); e $S_{10}$ - campo nativo $x$ milho $x$ arroz (SC). O delineamento experimental foi de blocos casualizados dispostos em parcelas subdivididas, com três repetições. As culturas de verão ocuparam as parcelas, enquanto as espécies de inverno foram alocadas nas subparcelas.

Os indicadores de sustentabilidade utilizados para avaliar as sucessões de culturas foram: (a) MS - matéria seca da parte aérea das espécies de inverno; (b) Mi/So - produção de grãos de milho ou de soja; (c) Arroz - produção de grãos de arroz; (d) Fert.1 - média harmônica dos valores de $\mathrm{pH}, \mathrm{MO}, \mathrm{P}$ e $\mathrm{K}$ obtidos na análise de solo ao final do experimento; (e) Fert.2 - média harmônica dos valores de Al, Ca, Mg obtidos na análise de solo ao final do experimento; (f) Fert.3 - média harmônica das medidas de resistência do solo à penetração, obtidas com um penetrômetro de impacto(IAA/ Planalsucar/Stolf), às profundidades (cm) de: 0-5; 5-10; 10-15; 15-20; 20-25; 25-30; 30-35; 3540; 40-45; (g) EP - energia produzida; (h) GL - ganho líquido de energia (EP - Energia Consumida); (i) VBP valor bruto da produção; (j) Rt. - rentabilidade (VBP/ Custo de Produção).

A EP e o GL foram quantificados utilizandose as orientações postuladas nos seguintes trabalhos: PIMENTEL (1980), EMBRAPA(1991), FREITAS et al. (1994), SANTOS et al. (2000, 2004) e PIMENTEL \& PATZEK (2005). O valor bruto da produção (VBP) e a rentabilidade das sucessões de culturas foram calculados considerando os custos médios relativos a insumos, a operações de campo e à venda de produtos.

A sustentabilidade das sucessões de culturas foi estimada com base em ELTUN et al. (2002), os quais estabelecem índices para padronizar as variáveis medidas, cada uma em relação àquela de melhor comportamento. Os subíndices componentes dos índices Fert. 1 e Fert. 2 foram obtidos em relação à condição inicial da área em estudo. Para resistência do solo à penetração, cada subíndice de Fert. 3 foi obtido dividindo-se o menor valor alcançado a cada profundidade, nas diversas sucessões, pelo valor da sucessão em estudo. Após calculada a média harmônica dos índices dos indicadores de cada sucessão, estas foram então comparadas entre si pelo Teste $t$, permitindo inferir-se sobre sua sustentabilidade.

$\mathrm{Na}$ discussão, os indicadores foram agrupados em quatro categorias de análise: a) Indicadores Agronômicos (MS, Milho/Soja, Arroz); b) Indicadores Ambientais (Fert.1, Fert.2, Fert. 3); c) Indicadores Energéticos (GL e EP); e d) Indicadores Econômicos (Rt. e VBP). 


\section{RESULTADOS E DISCUSSÃO}

Os indicadores de sustentabilidade para cada um dos sistemas de rotação e sucessão estudados estão na tabela 1. Nota-se que, enquanto a maioria dos valores mínimos de cada indicador esteve distribuída nos sistemas de cultivo que incluíram a cultura da soja, os maiores valores estiveram concentrados nas sucessões que contaram com a cultura do milho. Portanto, a expressão final desses sistemas de rotação e sucessão indicou índice de sustentabilidade maior para aqueles em que a cultura do milho esteve presente.

De outra forma, observando-se os pontos positivos e negativos de cada um dos sistemas, notouse que, mais do que os resultados representados pelo índice de sustentabilidade, que poderiam apontar um ou outro sistema como o melhor ou o mais equilibrado, os padrões de sustentabilidade são diferentes. Cada um dos sistemas de rotação e sucessão se apoiou, com maior ou menor importância, sobre uma ou mais das categorias de sustentabilidade consideradas. A hipótese geral é de que, quanto maior o equilíbrio entre as diferentes classes, melhor é o nível de sustentabilidade do sistema ou da atividade considerada (JARA, 1999).

Observa-se que os indicadores mais fortes do sistema $\mathrm{S}_{1}$ foram os agronômicos (MS, Soja eArroz), o energético (EP) e os econômicos (VBP e rentabilidade), todos com índices acima de 0,8 . Por outro lado, os indicadores Fert.1, Fert. 3 e GL foram os de menor expressão, ou seja, elementos que poderiam ser considerados fracos na análise dos sistemas testados. Isso significa que esse sistema de cultivo sustentouse, principalmente, sobre as categorias agronômica e econômica, tendo os aspectos ambientais menor contribuição. Em função dos elevados valores obtidos nos indicadores agronômicos, o sistema $\mathrm{S}_{1}$ apresentou a maior sustentabilidade entre os que utilizaram a cultura da soja.

Para o sistema $\mathrm{S}_{2}$, os principais indicadores também foram os indicadores agronômicos: soja e MS, Rt. e VBP. Os indicadores Fert. 1 e GL foram os elementos fracos desse sistema. A exemplo de $S_{1}$, esse sistema também se sustentou, principalmente, sobre as categorias agronômica e econômica. Apresentou a terceira maior sustentabilidade entre os que utilizaram a cultura da soja.

$\mathrm{O}$ sistema $\mathrm{S}_{3}$ não apresentou nenhum destaque $(>0,9)$ entre os indicadores de sustentabilidade. Entretanto, foi o que apresentou maior equilíbrio entre os valores, exceto para o indicador Fert. 1, que teve o maior valor. Por isso, alcançou o segundo mais alto índice médio de sustentabilidade entre todos os sistemas nos quais a cultura da soja participou. Pode-se inferir que o padrão de "ancoragem" de sustentabilidade do $\mathrm{S}_{3}$ esteve bem distribuído entre as quatro classes de indicadores considerados (agronômico, ambiental, energético e econômico).

Tabela 1 - Indicadores de sustentabilidade sobre as perspectivas agronômicas (MS, Mi/So e Arroz), ambientais (Fert. 1, Fert. 2 e Fert.3), energéticas (EP e GL) e econômicas (VBP e Rent.) e Índice de Sustentabilidade dos sistemas de rotação e sucessão de culturas. Pelotas, RS, 2007

\begin{tabular}{lccccccccccc}
\hline Sucessão de culturas $^{1}$ & MS $^{2}$ & M/S & Arroz & Fert.1 & Fert.2 & Fert.3 & EP & GL & VBP $^{\text {Rent. }}$ & Índice de Sustent. $^{3}$ \\
\hline $\mathrm{S}_{1}$ (PD) & 1,00 & 0,94 & 0,83 & 0,61 & 0,77 & 0,71 & 0,83 & 0,73 & 0,82 & 0,84 & 0,82 \\
$\mathrm{~S}_{2}$ (PD) & 0,83 & 0,98 & 0,78 & 0,62 & 0,76 & 0,76 & 0,74 & 0,63 & 0,78 & 0,79 & 0,78 \\
$\mathrm{~S}_{3}$ (PD) & 0,75 & 0,88 & 0,83 & 0,66 & 0,72 & 0,75 & 0,83 & 0,74 & 0,79 & 0,86 & 0,79 \\
$\mathrm{~S}_{4}$ (PD) & 0,74 & 0,95 & 0,81 & 0,59 & 0,82 & 0,66 & 0,73 & 0,60 & 0,79 & 0,74 & 0,76 \\
$\mathrm{~S}_{5}$ (SC) & 0,01 & 0,73 & 0,85 & 0,50 & 0,76 & 0,74 & 0,47 & 0,30 & 0,69 & 0,57 & 0,58 \\
$\mathrm{~S}_{6}$ (PD) & 1,00 & 0,83 & 0,87 & 0,55 & 0,77 & 0,74 & 0,98 & 0,93 & 0,94 & 0,95 \\
$\mathrm{~S}_{7}$ (PD) & 0,83 & 0,85 & 0,85 & 0,57 & 0,70 & 0,73 & 0,97 & 0,93 & 0,99 & 0,97 \\
$\mathrm{~S}_{8}$ (PD) & 0,75 & 1,00 & 0,87 & 0,65 & 0,77 & 0,71 & 1,00 & 0,96 & 0,92 & 0,98 & 0,87 \\
$\mathrm{~S}_{9}$ (PD) & 0,74 & 0,90 & 0,83 & 0,60 & 0,82 & 0,80 & 0,90 & 0,80 & 0,91 & 0,86 \\
$\mathrm{~S}_{10}$ (SC) & 0,01 & 0,73 & 1,00 & 0,41 & 0,63 & 0,75 & 0,67 & 0,54 & 0,87 & 0,73 & 0,83 \\
\hline
\end{tabular}

Teste F para o Índice de Sustentabilidade - **; CV - 9,31\%.

${ }^{1} \mathrm{~S}_{1}$ - Gramínea x soja x arroz (PD); $\mathrm{S}_{2}$ - Leguminosa x soja x arroz (PD); $\mathrm{S}_{3}$ - Consórcio x soja x arroz (PD); $\mathrm{S}_{4}$ - Nabo x soja x arroz (PD); $\mathrm{S}_{5}$ - Campo nativo x soja x arroz (SC); $\mathrm{S}_{6}$ - Gramínea x milho x arroz (PD); $\mathrm{S}_{7}$ - Leguminosa x milho x arroz (PD); $\mathrm{S}_{8}$ - Consórcio x milho x arroz (PD); $\mathrm{S}_{9}$ - Nabo x milho x arroz (PD); $\mathrm{S}_{10}$ - Campo nativo x milho x arroz (SC).

${ }^{2}$ MS - matéria seca das espécies de inverno; Mi/So - rendimento de grãos (milho ou soja); Arroz - rendimento de grãos de arroz; Fert.1 média harmônica dos valores de Al, Ca, Mg; Fert.2 - média harmônica dos valores de pH, MO, P e K; Fert.3 - média harmônica das medidas de resistência a penetração do solo, obtidas com o penetrômetro de impacto; EP - energia produzida; GL - ganho líquido de energia; VBP valor bruto da produção; Rent. - rentabilidade.

${ }^{3}$ Máximo $=1 ;$ mínimo $=0$ 
$\mathrm{O}$ sistema $\mathrm{S}_{4}$ apresentou como destaque, entre seus indicadores, a produção de grãos de soja, seguida pela produção de grãos de arroz e, logo após, pelo indicador ambiental Fert. 2. Este atingiu o maior valor entre todas as sucessões consideradas e foi igual ao obtido no sistema $\mathrm{S}_{9}$. No entanto, esse sistema apresentou o pior índice para o indicador Fert. 3, que está relacionado à resistência do solo à penetração. Em relação a esse indicador, esperava-se que fosse destaque, já que faz parte do sistema a cultura do nabo, considerada como “arado/descompactador biológico”. Outro ponto de pouca contribuição desse sistema refere-se ao GL, que apresentou o segundo menor valor entre os demais. Em função do exposto, esse sistema apresentou a menor sustentabilidade entre os que utilizam soja em PD.

O sistema $S_{5}$, que utiliza o sistema convencional de cultivo, apresentou os mais baixos valores para os indicadores agronômicos (MS e Mi/ So), energéticos (EP e GL) e econômicos (VBP e Rt.) entre todos os sistemas, conferindo-lhe a menor sustentabilidade entre todos os sistemas analisados, mesmo entre os que incluem a cultura do milho.

O maior índice de sustentabilidade para os sistemas que incluem a cultura da soja, observado em $\mathrm{S}_{1}$, caracteriza bem a importância de se alternarem espécies com habilidades diferenciadas, seja no quesito de aproveitamento de nutrientes do solo, como no de resistência ou na suscetibilidade a doenças ou pragas (SÁ, 1993; SANTOS \& REIS 2001).

$\mathrm{O}$ sistema $\mathrm{S}_{6}$ apresentou valores elevados $(>0,9)$ para os índices MS, EP, GL, VBPe Rt, resultando no maior índice médio de sustentabilidade. Entretanto, esse sistema apresentou os seus pontos fracos nos indicadores ambientais Fert.1, Fert.3 e Fert. 2, com os piores índices. Portanto, esse sistema não apresentou uma distribuição equilibrada entre todas as categorias consideradas.

Os pontos fortes do sistema $\mathrm{S}_{7}$ foram os indicadores energéticos (EP e GL) e os econômicos (VBP e Rt.), todos com índices superiores a 0,9. À semelhança do sistema anterior, os pontos de menor contribuição foram os indicadores ambientais. Mesmo assim, esse sistema apresentou o segundo melhor índice médio de sustentabilidade. Como no sistema anterior, o sistema $\mathrm{S}_{7}$ não apresenta uma distribuição equilibrada entre as classes consideradas.

$\mathrm{O}$ sistema $\mathrm{S}_{8}$ apresentou, juntamente com o $\mathrm{S}_{6}$, os maiores índices médios de sustentabilidade. Isso ocorreu em razão da presença de quatro indicadores com registros máximos relativos: Mi/So, EP, GL, e Rt. e do indicador VBP, o qual, embora não tenha apresentado os maiores valores, apareceu em destaque $(>0,9)$. Os pontos fracos desse sistema foram relacionados à MS e aos indicadores ambientais. Entretanto, os registros observados para estes estiveram entre os melhores, o que colaborou para obtenção do escore de sustentabilidade. Embora o índice médio de sustentabilidade tenha sido igual ao do sistema $\mathrm{S}_{6}$, pode-se inferir que esse sistema foi o mais sustentável, já que apresentou melhor distribuição e equilíbrio entre os diversos aspetos considerados, o que está de acordo com as postulações de QUIROGA (2002).

O sistema $\mathrm{S}_{9}$ apresentou o pior desempenho para sustentabilidade entre os que utilizaram milho em plantio direto, mas com registro ligeiramente superior ao observado para o melhor sistema que incluiu a cultura da soja. Os indicadores ambientais Fert.2 e Fert.3 destacaram-se entre todos os sistemas estudados. O bom desempenho do indicador Fert. 3 no sistema $\mathrm{S}_{9}$ já era esperado devido à participação do nabo. Os demais indicadores apresentaram valores médios considerados bons, o que determinou um bom índice médio de sustentabilidade a esse sistema.

$\mathrm{O}$ sistema $\mathrm{S}_{10}$, conduzido no sistema convencional de cultivo, a exemplo do que aconteceu com a soja, também apresentou o pior desempenho no que se refere à sustentabilidade entre os sistemas que incluíram o milho. O índice médio de sustentabilidade desse sistema foi inferior ao verificado em todos aqueles em que a soja foi conduzida em plantio direto. No entanto, o $S_{10}$ apresentou o maior registro para o indicador agronômico arroz. Por outro lado, também foi o que apresentou os mais baixos registros para os indicadores agronômicos MS, Mi/So e para os indicadores ambientais Fert.1 e Fert.2, o que explica o baixo índice médio de sustentabilidade estimado para esse sistema.

Houve diferença significativa entre os índices de sustentabilidade dos sistemas de rotação e sucessão de culturas. Os sistemas $\mathrm{S}_{6}$ e $\mathrm{S}_{8}$ foram os que apresentaram os maiores índices de sustentabilidade $(0,86)$, seguidos pelos sistemas $S_{7}(0,84), S_{9}(0,82)$, ambos em plantio direto e que incluíram milho. Essa superioridade se deve, em parte, à presença do milho, cultura com alta capacidade de produção de resíduos, já que o teor de nutrientes do solo é afetado, entre outras formas, pela quantidade de material vegetal que retorna ao solo (CASTRO \& MARIA, 1993).

Em relação aos sistemas que incluíram a cultura da soja, o melhor índice de sustentabilidade encontrado foi no $S_{1}(0,81)$, seguido pelos sistemas $S_{3}$ $(0,78), S_{2}(0,77)$ e $S_{4}(0,74)$, também todos em plantio direto, o que está em acordo com os resultados de SANTOS et al. (2006).

A análise de contrastes entre os índices de sustentabilidade pelo teste $\mathrm{t}(\mathrm{P}<0,05)$ indicou que as comparações uma a uma entre os sistemas que incluíram a cultura da soja apresentaram diferença significativa, sempre que se compararam as conduzidas em $\mathrm{PD}\left(\mathrm{S}_{1}\right.$, $\mathrm{S}_{2}, \mathrm{~S}_{3}$ e $\mathrm{S}_{4}$ ) com as em SC $\left(\mathrm{S}_{5}\right)$ (Tabela 2). Também foi

Ciência Rural, v.39, n.6, set, 2009. 
Tabela 2 - Matriz de comparações entre os índices de sustentabilidade dos diferentes sistemas de rotação e sucessão de culturas pelo teste t $(\mathrm{P}=0,05 \%)$. Pelotas, RS, 2007.

\begin{tabular}{|c|c|c|c|c|c|c|c|c|c|c|}
\hline Sucessão de culturas & $\mathrm{S}_{1}$ & $\mathrm{~S}_{2}$ & $\mathrm{~S}_{3}$ & $\mathrm{~S}_{4}$ & $\mathrm{~S}_{5}$ & $\mathrm{~S}_{6}$ & $\mathrm{~S}_{7}$ & $\mathrm{~S}_{8}$ & $\mathrm{~S}_{9}$ & $\mathrm{~S}_{10}$ \\
\hline $\mathrm{S}_{1}$ - Gramínea x soja x arroz (PD) & - & ns & ns & $*$ & $*$ & $*$ & ns & $*$ & ns & $*$ \\
\hline $\mathrm{S}_{2}$ - Leguminosa $\mathrm{x}$ soja $\mathrm{x}$ arroz (PD) & & - & ns & ns & $*$ & $*$ & $*$ & $*$ & $*$ & * \\
\hline $\mathrm{S}_{3}$ - Consórcio x soja x arroz (PD) & & & - & ns & $*$ & $*$ & $*$ & $*$ & ns & $*$ \\
\hline $\mathrm{S}_{4}-$ Nabo x soja x arroz (PD) & & & & - & $*$ & $*$ & $*$ & $*$ & $*$ & $*$ \\
\hline $\mathrm{S}_{5}-$ Campo nativo $\mathrm{x}$ soja $\mathrm{x}$ arroz (SC) & & & & & - & $*$ & $*$ & $*$ & $*$ & $*$ \\
\hline $\mathrm{S}_{6}$ - Gramínea x milho x arroz (PD) & & & & & & - & ns & ns & ns & $*$ \\
\hline $\mathrm{S}_{7}-$ Leguminosa $\mathrm{x}$ milho $\mathrm{x}$ arroz (PD) & & & & & & & - & ns & ns & $*$ \\
\hline $\mathrm{S}_{8}$ - Consórcio $\mathrm{x}$ milho $\mathrm{x}$ arroz (PD) & & & & & & & & - & ns & $*$ \\
\hline $\mathrm{S}_{9}-$ Nabo x milho x arroz (PD) & & & & & & & & & - & $*$ \\
\hline $\mathrm{S}_{10}$ - Campo nativo x milho $\mathrm{x}$ arroz (SC) & & & & & & & & & & - \\
\hline
\end{tabular}

ns - não significativo; * $\mathrm{P}=(0,05)$.

significativa a diferença entre os índices de sustentabilidade dos sistemas $\mathrm{S}_{1}$ e $\mathrm{S}_{4}$.

As diferenças entre os índices de sustentabilidade de $\mathrm{S}_{1} \times \mathrm{S}_{7}, \mathrm{~S}_{1} \times \mathrm{S}_{9}$ e $\mathrm{S}_{3} \times \mathrm{S}_{9}$ não foram significativas. Todas as demais diferenças possíveis envolvendo soja versus milho foram significativas.

Dentre os sistemas com milho em PD, as diferenças entre os índices de sustentabilidade não foram significativas. Entretanto, quando se comparou os sistemas conduzidos em PD (S6, S7, S8 e S9) com os conduzidos no sistema convencional (SC), as diferenças que se verificaram foram significativas.

Um destaque salientado pelo cálculo dos índices foi a maior sustentabilidade conferida a qualquer sistema conduzido em $\mathrm{PD}$, quando comparado com os conduzidos em SC, tanto nos sistemas que envolviam a cultura do milho, quanto os que envolviam a cultura da soja. Segundo ACEVEDO \& SILVA(2003), uma baixa sustentabilidade está geralmente associada ao SC, enquanto o PD tem vários impactos ambientais positivos (VAN RAIJ, 2006). PECHE FILHO (2007) postula que o sistema de plantio direto é a melhor forma de se "agricultar sustentavelmente”.

\section{CONCLUSÕES}

Todos os sistemas de rotação e sucessão de culturas que incluem milho apresentam um maior índice de sustentabilidade. Os sistemas $\mathrm{S}_{1}$ [gramínea $\mathrm{x}$ soja $x$ arroz (PD)] e $S_{4}$ [nabo $x$ soja $x$ arroz (PD)] apresentam, respectivamente, a maior e a menor sustentabilidade entre aqueles sistemas em que a cultura da soja participa. O sistema de rotação e sucessão $\mathrm{S}_{5}$ [campo nativo x soja x arroz (SC)] apresenta o menor índice de sustentabilidade. O sistema de rotação e sucessão $\mathrm{S}_{8}$ [consórcio $\mathrm{x}$ milho $\mathrm{x}$ arroz (PD)] apresenta a melhor distribuição e o melhor equilíbrio entre as diversas classes de sustentabilidade consideradas. $\mathrm{O}$ sistema de rotação e sucessão $\mathrm{S}_{10}$ [campo nativo x milho $\mathrm{x}$ arroz (SC)] tem o pior desempenho, no que se refere à sustentabilidade, entre aqueles que incluíram a cultura do milho. O sistema de PD confere maior sustentabilidade aos sistemas de rotação e sucessão de culturas.

\section{REFERÊNCIAS}

ACEVEDO, E.; SILVA, P. Sistema de labranza y sustentabilidad agrícola en cultivos anuales. Simiente, v.74, n.3-4, p.3-6 .2003. Disponível em: <http://www.sap.uchile.cl/Archivos/ea/ $\mathrm{S}$ i s t e m a \% $20 \mathrm{de} \% 20 \mathrm{~L}$ a b r a n z a \% 20 y \% 20 sustentabilidad\%20Agr\%EDcola\%20en\%20Cultivos 1.pdf> Acesso em: 07 ago. 2007.

BAILEY, A.P. et al. A comparison of energy use in conventional and integrated arable farming systems in the UK. Agriculture, Ecosystems and Environment, v.97, p.241-253, 2003 Disponível em: <http:// www.sciencedirect.com/science?_ob=ArticleURL\&_udi=B6T3Y488 G 5 G V- $4 \&$ \&_us e r $=5889052 \&$ \&_cover Date $=$ $07 \% 2$ F $31 \% 2$ F 2003 \&_a li d = 8 88846212 \&

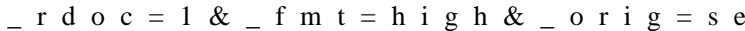
arch\&_cdi $=4959 \&$ \& sort $=$ d \&_docanchor $=\&$ view $=$ c \&_ct $=1 \&_{-}$a c ct $=$C $000012878 \&$ \& version $=1 \&$ _ url Version $=0 \&$ _ u s e rid $=5889052 \&$ m d $5=$ 8be4ace36d45143d18005cc03accb60b>. Doi:10.1016/S01678809(03)00115-4.

CAMPOS, A.T.; CAMPOS, A.T. Balanços energéticos agropecuários: uma importante ferramenta como indicativo de sustentabilidade de agroecossistemas. Ciência Rural, v.34, n.6, p.1977-1985. 2004. Disponível em: http://www.scielo.br/ scielo.ph p ? s c ri pt = sci_art text \& pid = S 0103 84782004000600050\&lng=pt\&nrm=iso. Doi: $10.1590 /$ S0103-84782004000600050.

CASTRO, O.M. de, DE MARIA, I.C. Fósforo, potássio e matéria orgânica em um latossolo roxo, sob sistemas de manejo com milho e soja. Revista Brasileira Ciência do Solo, v.17, n.3, p.471-477, 1993.

Ciência Rural, v.39, n.6, set, 2009. 
ELTUN, R. et al. A comparison of environmental, soil fertility, yield, and economical effects in six cropping systems based on an 8-year experiment in Norway. Agriculture, Ecosystems and Environment, v.90, n.2, p.155-168, 2002. Disponível em: <http:/ /www.sciencedirect.com/science?_ob=ArticleURL\&_udi=B6T3Y4606SBX-F\&_user=5889052\&_coverDate=>. Acesso em: 20 mai 2006. Doi:10.1016/S0167-8809(01)00198-0.

EHLERS, E. Agricultura sustentável: origens e perspectivas de um novo paradigma. 2.ed. Guaíba: Agropecuária, 1999. 157p.

EMBRAPA. Centro Nacional de Pesquisa de Suínos e Aves (Concórdia, SC). Tabela de composição química e valores energéticos de alimentos para suínos e aves. 3.ed. Concórdia, 1991. 97p. (EMBRAPA-CNPSA. Documentos, 19).

FREITAS, E.A.G. de et al. Tabela de composição químicobromatológica e energética dos alimentos para animais ruminantes em Santa Catarina. Florianópolis: Epagri, 1994. 33p. (Epagri. Documentos, 155).

GOMES. A da S. et al. Caracterização de Indicadores da qualidade do solo, com ênfase às áreas de várzea do Rio Grande do Sul. Pelotas: Embrapa Clima Temperado, 2006. 40p. (Embrapa Clima Temperado. Documentos, 169).

JARA, C. J. Sustentabilidade: uma encruzilhada civilizatória. In: NETO, A. B. (Org.) Sustentabilidade e cidadania: o papel da extensão rural. Porto Alegre: Emater, 1999. 208 p.

LAL, R. Métodos para avaliação do uso sustentável dos recursos solo e água nos trópicos. Jaguariúna: Embrapa Meio Ambiente, 1999. 97p. (Documentos 03).

LOPES, S. B.; ALMEIDA, J. Metodologia para análise comparativa de sustentabilidade em sistemas agroflorestais / Methodology for comparative analysis of sustainability in agroforestry sistems. Revista de Economia e Sociologia Rural, v.41, n.1, p.79-109, 2002.

MASERA, O. et al. Sustentabilidad y manejo de recursos naturales. El marco de evaluación MESMIS. Mexico: Mundi-Prensa Mexico, 1999. 110p.

PECHE FILHO, A. A gestão do sistema de plantio direto na filosofia da qualidade total. 2007. Artigo em Hypertexto. Disponível em: <http://www.infobibos.com/ Artigos/2007_1/QT/Index.htm>. Acesso em: 20 mar 2007

PIMENTEL, D. Energy inputs for the production, formulations, packaging and transport for various pesticides. In: PIMENTEL, D. (ed.). Handbook of energy utilization in agriculture. Boca Raton: CRC, 1980. p.45-48

PIMENTEL, D.; PATZEK, T.W. Ethanol production using corn, switchgrass, and wood; biodiesel production using soybean and sunflower. Natural Resources Research, v.14,n.1, p.6576, 2005. Disponível em: <http:// www.springerlink.com.w10106.dotlib.com.br/content/ r1552355771656v0/fulltext.pdf >. Acesso em: 20 mar 2007. Doi: $10.1007 / \mathrm{s} 11053-005-4679-8$.
PINTO, L.F.S. et al. Caracterização de solos de várzea. In: GOMES, A. da S.; PAULETTO, E.A. Manejo do solo e da água em áreas de várzea. Pelotas: Embrapa Clima Temperado, 1999. p.11-36.

QUIROGA, R. Información y participación em el desarollo de la sustentabilidad en America Latina. La transicion hacia el desarrollo sostenible. Mexico: Instituto Nacional de Ecología, Universidad Autónoma Metropolitana (UAM) y PNUMA, 2002. 578p.

SÁ, J.C.de M. Manejo da fertilidade do solo no plantio direto. Castro: Fundação ABC, 1993. 96p.

ROYAL SOCIETY OF CHEMISTRY. The vital ingredient: chemical science and engineering for sustainable food. January 2009. Disponível em: <http://www.rsc.org/images/ FoodReport_tcm18-142397.pdf>. Acesso em: 28 jan 2009.

SANTOS, H.P. dos; REIS, E.M. Rotação de culturas em plantio direto. Passo Fundo: Embrapa Trigo, 2001. 212p.

SANTOS, H.P. et al. Sistemas de produção de grãos com pastagens anuais de inverno e de verão, sob plantio direto. Passo Fundo: Embrapa Trigo, 2004. 39p. (Embrapa Trigo. Documentos Online; 45). Disponível em: http:// www.cnpt.embrapa.br/biblio/do/p_do45.htm>. Acesso em 14 mar 2005.

SANTOS, H.P. et al. Conversão energética e balanço energético de sistemas de rotação de culturas para triticale. Ciência Rural, v.30, n.1, p.43-48, 2000. Disponível em: <http://www.scielo.br/ s cielo.php ? s c ri pt = s ci_art text \& pid = S 0103 84782000000100007\&lng=pt\&nrm=iso $>$. Acesso em: $20 \mathrm{mar}$ 2007. Doi: 10.1590/S0103-84782000000100007.

SANTOS, H.P. dos et al. Rendimentos de grãos de soja em função de diferentes sistemas de manejo de solo e de rotação de culturas. Ciência Rural, v.36, n.1, p.21-29, 2006. Disponível em: <http:/ /www.scielo.br/scielo.php?script=sci_arttext\&pid=S010384782006000100004\&lng=pt\&nrm=iso $>$. Acesso em: $20 \mathrm{mar}$ 2007. Doi: 10.1590/S0103-84782006000100004.

VAN RAIJ, B. Plantio direto e desenvolvimento sustentável. Disponível em: <http://www.agrisus.org.br/artigos/ pd.sustentavel.htm> Acesso em 6/10/2006.

VERNETTI JR, F. de J. et al. Plantio direto de soja e milho em solo de várzea e em seqüência a diferentes coberturas mortas. In: DA SILVA, C.A.S.; GASTAL, M.F da C. (Eds.). REUNIÃO TÉCNICA, DIVERSIFICAÇÃO DO USO DE VÁRZEAS DE CLIMA TEMPERADO, 2002, Pelotas. Anais... Pelotas: Embrapa Clima Temperado, 2002. p. 153-157. (Embrapa Clima Temperado. Documentos, 90)

VERNETTI JUNIOR, F. de J. et al. Arroz irrigado em sucessão a milho e soja. In: CONGRESSO BRASILEIRO DE ARROZ IRRIGADO, 3; REUNIÃO DA CULTURA DE ARROZ IRRIGADO, 25., 2003, Balneário Camboriú, SC. Anais... Itajaí: EPAGRI, 2003. p.246-247. 